\title{
Comparison between Abbott m2000 RealTime and Alinity m STI systems for detection of Chlamydia trachomatis, Neisseria gonorrhoeae, and Mycoplasma genitalium
}

\author{
Björn Herrmann ${ }^{1,2}$ (1) Karin Malm ${ }^{1}$ \\ Received: 16 October 2020 / Accepted: 16 December 2020 / Published online: 15 March 2021 \\ (C) The Author(s) 2020
}

\begin{abstract}
The new Abbott Alinity m STI Assay was compared with Abbott m2000 RealTime PCR. For Chlamydia trachomatis, 26 (7.5\%) of 347 samples were positive in the Alinity assay and 24 (6.9\%) in the m2000 assay. Corresponding figures for Neisseria gonorrhoeae were 23 (6.6\%) and 17 (4.9\%). For Mycoplasma genitalium, 22 (7.9\%) of 279 samples were positive in the Alinity assay and $18(6.5 \%)$ in the $\mathrm{m} 2000$ assay, for which DNA extraction was performed on an m2000sp instrument combined with in-house real-time PCR. The Alinity assay has at least the same sensitivity as the $\mathrm{m} 2000$ assay. The specificity was evaluated by discrepancy analysis.
\end{abstract}

Keywords Chlamydia trachomatis $\cdot$ Neisseria gonorrhoeae $\cdot$ Mycoplasma genitalium $\cdot$ Nucleic acid amplification tests

Over the last three decades, continuous advances have been made in the molecular diagnostics of bacteria causing sexually transmitted infections. Diagnostic accuracy has improved, automation has increased, and multiplex detection of target organisms is now common. In the last two decades, detection of Chlamydia trachomatis (CT) and Neisseria gonorrhoeae (NG) has typically been performed as a duplex test in the major commercial assays, with Mycoplasma genitalium (MG) now added to such assays. Although not relevant for all countries and settings, the parasite Trichomonas vaginalis is also included.

The Abbott Alinity platform is a new random-access system developed to replace the m2000 RealTime platform. In this study, the Alinity m STI Assay was compared with the Abbott m2000 RealTime System for detection of CT/NG, and for MG with DNA extraction on Abbott m2000sp (as for CT/ NG) and detection by an adapted in-house real-time PCR [1]. In the in-house real-time PCR, $10 \mu \mathrm{L}$ DNA extract from the

Björn Herrmann

bjorn.herrmann@medsci.uu.se

1 Department of Clinical Microbiology, Uppsala University Hospital, SE-751 85 Uppsala, Sweden

2 Section of Clinical Bacteriology, Department of Medical Sciences, Uppsala University, Uppsala, Sweden $\mathrm{m} 2000$ procedure was used. This combination of DNA extraction on $\mathrm{m} 2000$ and in-house PCR detection is referred to here as "m2000 assay" for MG. Samples were also analyzed for Trichomonas vaginalis in the Alinity assay.

Duplicate samples from urine (153), cervix/vagina (130), rectum (59), or throat (5) were taken from persons attending an STI clinic for suspected CT/NG/MG infection. Abbott multi-Collect Specimen Collection Kits were used for the m2000 RealTime System and Alinity-m multi-Collect Specimen Collection Kits for the Alinity-m STI. Samples were taken from consecutive patients and the sample order for the two collection kits was shifted every week to avoid bias.

In our laboratory, Alinity NG-positive samples are always tested by a confirmatory duplex in-house PCR using opa and porA as target genes [2]. Since in the Alinity system the DNA eluate is used directly for detection, with no leftover volume for additional tests, a separate DNA extraction had to be used for confirmatory PCR. This was done using a $200 \mu \mathrm{L}$ sample diluted in $200 \mu \mathrm{L}$ nuclease-free water for DNA extraction in a magLEAD-12gC extraction robot (Precision System Science, Japan). In the confirmatory PCR, $5 \mu \mathrm{L}$ eluate was analyzed.

Evaluation of $\mathrm{NG}$ and $\mathrm{MG}$ detection was performed on all Alinity-positive samples over a 6-month period, including clinical data and antibiotic treatment. From all Alinity MGpositive samples, DNA was extracted with magLEAD and retested with PCR (Diagenode S-DiaMGRes-kit, Diagenode Diagnostics, Liège, Belgium). 
The performance of the Alinity-m STI system was also tested on proficiency panels. For CT/NG, the QCMD 2020 DNA EQA panels QAB004101_2 and QAB034126_2 https:// qcmd.org were used, and for $\mathrm{MG}$ panel 2019-288 from the quality assessment organization EQUALIS https://www. equalis.se/en/about-us/.

The data used in this study were part of routine practices in the hospital prior to and after implementation of a new method (see supplement). Data on antimicrobial treatment were taken from case records. The study was approved by the Regional Ethical Review Board in Uppsala, Sweden (ref. 2020-04572).

Analysis of CT/NG was performed on 347 samples. For CT, 26 samples $(7.5 \%)$ were positive in the Alinity assay and 24 $(6.9 \%)$ in the $\mathrm{m} 2000$ assay. Corresponding figures for NG were 23 (6.6\%) and 17 (4.9\%); see Table 1. The number of detected cases was not statistically different for either $\mathrm{CT}$ or $\mathrm{NG}$ when the two assays were compared. Using the $\mathrm{m} 2000$ assay as reference, the Alinity assay for CT detection had a sensitivity of $95.8 \%$ (78.9-99.9) and a specificity of $99.1 \%$ (97.3-99.8). For NG the corresponding figures were $100 \%(80.5-100.0)$ and $98.2 \%$ (96.1-99.3). Agreement for the two assays was $98.8 \%$ (97.199.7, Table 1) for CT and 98.3\% (97.1-99.7) for NG. The distribution of samples by different types is shown in Table 2 .

The six NG samples that were Alinity-positive/m2000negative had cycle of threshold $(\mathrm{Ct})$ values ranging from 22.2 to $38.9(22.2 ; 24.4 ; 24.7 ; 35.6 ; 37.0 ; 38.9)$. By mistake these six samples were not tested for discrepancy analysis with the confirmatory duplex in-house PCR by Goire et al. [2]. However, of 67 Alinity NG-positive samples over 6 months in our laboratory, 51 were unequivocally confirmed in the duplex PCR, with detection of both targets. Of the remaining 16 samples, four were interpreted as representing true NG infection, one of which was negative for the porA target; see supplement. Nine samples were determined as NG trace DNA after antibiotic treatment, with a mean $\mathrm{Ct}$ value of 36.7 in Alinity (range 35.1-39.5), and three as an unspecific reaction, with a mean Ct value of 36.4 in Alinity (range 33.938.9). Thus, Alinity-positive samples that could not be confirmed by in-house PCR indicate low copy numbers of the target, leading to discrepant results.
Analysis of MG was performed on 279 samples (a subset of the 347 samples), of which $22(7.9 \%)$ were positive in the Alinity assay and $18(6.5 \%)$ in the $\mathrm{m} 2000$ assay. The five samples that were Alinity-positive/m2000-negative had cycle numbers in the range 35.7-39.8, probably indicating higher sensitivity for Alinity. Agreement for the two assays was 97.8\% (95.4-99.2).

Over 6 months, 167 Alinity MG-positive samples were retested with an additional PCR (Diagenode) and 159 were positive in both assays. The remaining eight samples had a mean Ct value of 34.7 (31.9-37.5) in Alinity and were negative in the Diagenode assay.

In 347 samples, $T$. vaginalis was not detected in the Alinity assay. This was expected, since the prevalence of this agent is almost zero in Sweden and detection is confined to sporadic imported cases [3].

In the external quality assessment panels of five samples for CT and NG, the Alinity assay had expected detection results for both bacteria in all samples, with $\mathrm{Ct}$ values between 24.9 and 35.8 (Supplementary table 1). Analysis of the MG panel produced a similar outcome: all six samples gave expected results in the Alinity assay, with a $\mathrm{Ct}$ value range of 30.5-38.2. A point to be noted was that the weakest positive sample in the Alinity assay was negative when the same panel was tested in the m2000 assay. Of the 33 participating laboratories, $18 \%$ did not detect $M G$ in that sample.

This comparative study was undertaken with the objective of replacing the $\mathrm{m} 2000$ platform with the next-generation platform Alinity. One strength of the study is that it was performed in a clinical laboratory without additional precautions beyond those routinely applied, i.e., the outcome reflects what is achieved in a real-world laboratory setting. The study design also had limitations. First, the number of tests did not achieve sufficient statistical power to detect any differences in sensitivity between the Alinity and m2000 assays for CT/ NG detection. Nevertheless, our results indicate that the Alinity-m STI assay had at least the same sensitivity as the m2000 assay for CT/NG detection. Since MG is not included in the Abbott m2000 assay, comparison between the Alinity assay and a combination of DNA extraction on m2000 and an
Table 1 Analysis of 347 duplicate samples with suspected CT/NG-infection collected in Abbott multi-collect m2000 tubes and in Alinity m STI tubes. Analyzed with Abbott m2000 and Alinity m

\begin{tabular}{llll}
\hline & $\begin{array}{l}\text { C. trachomatis } \\
\text { No. of samples }\end{array}$ & $\begin{array}{l}\text { N. gonorrhoeae } \\
\text { No. of samples }\end{array}$ & $\begin{array}{l}\text { M. genitalium } \\
\text { No. of samples }\end{array}$ \\
\hline m2000 positive/Alinity m positive & 23 & 17 & 17 \\
m2000 negative/Alinity m positive & 3 & 6 & 5 \\
m2000 positive/Alinity m negative & 1 & 0 & 1 \\
m2000 negative/Alinity m negative & 320 & 324 & 256 \\
Agreement \% & 347 & 347 & 279 \\
Kappa value & $98.8(97.1-99.7)$ & $98.3(96.3-99.4)$ & $97.8(95.4-99.2)$ \\
& $0.914(0.830-0.998)$ & $0.841(0.717-0.966)$ & $0.839(0.712-0.965)$ \\
\hline
\end{tabular}


Table 2 Distribution of sample types analyzed with Alinity $\mathrm{m} \mathrm{STI}^{\mathrm{a}}$
C. trachomatis
N. gonorrhoeae
M. genitalium

Specimen type No. of samples No. of positive No. of negative No. of positive No. of negative No. of samples No. of positive No. of negative

\begin{tabular}{lllllllll}
\hline Urine & 153 & 6 & 147 & 8 & 145 & 123 & 9 & 114 \\
Throat & 5 & 1 & 4 & 2 & 3 & 0 & 0 & 0 \\
Cervix/vagina & 130 & 13 & 117 & 8 & 122 & 111 & 12 & 99 \\
Rectum & 59 & 6 & 53 & 5 & 54 & 45 & 1 & 44 \\
& 347 & 26 & 321 & 23 & 324 & 279 & 22 & 257 \\
\hline
\end{tabular}

${ }^{a}$ All samples also analyzed with the m2000 assay, but the low number of different positive sample types did not allow relevant statistical analysis for comparison

in-house PCR method is not straightforward. But the outcome for clinical samples and the proficiency panel indicates that the Alinity assay has sufficient detection capacity to be used. Since the copy number of MG is up to a hundred times lower in clinical samples compared to CT [4], test sensitivity is essential for detection of MG.

Another limitation of our study is that the number of specific sample types did not permit separate analysis for urine, cervical/vaginal, rectal, and throat specimens. According to the package insert for the Alinity-m STI AMP Kit (version 53-608074/R1), the test is only validated for samples from the cervix/vagina and urine for $\mathrm{CT} / \mathrm{NG}$ and only for endocervical specimens from for MG. Table 2 indicates that detection is entirely possible from all specimen types, although the throat is rarely a clinically relevant target for MG [5].

A technical limitation of the automated Alinity system with integrated DNA extraction and target detection is that it does not permit downstream nucleic acid amplification tests. Confirmation of NG, which is needed in low-prevalence settings [6], and antibiotic resistance detection for MG therefore require separate DNA extraction from the Alinity-m multicollect specimen sample tube (09N19-001). This sample tube contains different components, including lysing agents. Efficient DNA extraction in other systems is consequently often suboptimal, since the compounds from different systems are mixed and disturb the balance of components. To avoid clogging, in our laboratory we dilute the sample from the Alinity tube twofold in water before DNA extraction in the magLEAD- $12 \mathrm{gC}$ extraction robot.

Evaluation of nucleic acid amplification tests for CT/NG has previously been controversial, and their performance has been questioned even after careful discrepancy testing [7, 8]. On the other hand, well-established commercial test systems provide high enough sensitivity for what is clinically relevant and may even be so sensitive that contamination of samples may lead to false-positive results [9-11]. Specificity has been a major problem for some amplification tests regarding NG, but the newest generation of test systems have acceptable performance [12], although not in low-prevalence settings where confirmation tests are needed to avoid false-positive results [13].

In summary, the Alinity-m STI system provides a userfriendly automatic system with adequate detection of CT, NG, and MG.

Supplementary Information The online version contains supplementary material available at https://doi.org/10.1007/s10096-020-04135-9.

Data availability (data transparency) Available on request.

Authors' contributions Björn Herrmann designed the study. Both authors contributed to data collection and analysis. The first draft of the manuscript was written by Björn Herrmann. Karin Malm contributed on previous versions of the manuscript and both authors read and approved the final manuscript.

Funding Open Access funding provided by Uppsala University. The work was financed as part of the ordinary work of the hospital laboratory.

\section{Compliance with ethical standards}

Conflict of interest The authors declare that they have no conflicts of interest.

Ethics approval (include appropriate approvals or waivers) The study was approved by the Regional Ethical Review Board in Uppsala, Sweden (ref. 2020-04572).

Consent to participate (include appropriate statements) Not applicable.

Consent for publication (include appropriate statements) Not applicable.

Code availability (software application or custom code) Not applicable.

\section{References}

1. Jensen JS, Björnelius E, Dohn B, Lidbrink P (2004) Use of TaqMan 5 ' nuclease real-time PCR for quantitative detection of Mycoplasma genitalium DNA in males with and without urethritis who were 
attendees at a sexually transmitted disease clinic. J Clin Microbiol 42:683-692. https://doi.org/10.1128/jcm.42.2.683-692.2004

2. Goire N, Nissen MD, LeCornec GM, Sloots TP, Whiley DM (2008) A duplex Neisseria gonorrhoeae real-time polymerase chain reaction assay targeting the gonococcal porA pseudogene and multicopy opa genes. Diagn Microbiol Infect Dis 61:6-12. https://doi.org/10.1016/j.diagmicrobio.2007.12.007

3. Pellrud H, Golparian D, Nilsson CS, Falk M, Fredlund H, Unemo M (2015) Trichomonas vaginalis infections are rare among young patients attending an STI clinic in Sweden. Acta Derm Venereol 95: 343-344. https://doi.org/10.2340/00015555-1946

4. Walker J, Fairley CK, Bradshaw CS, Tabrizi SN, Chen MY, Twin J, Taylor N, Donovan B, Kaldor JK, McNamee K, Urban E, Walker S, Currie M, Birden H, Bowden F, Gunn J, Pirotta M, Gurrin L, Harindra V, Garland S, Hocking JS (2011) The difference in determinants of Chlamydia trachomatis and Mycoplasma genitalium in a sample of young Australian women. BMC Infect Dis 11:35. https://doi.org/10.1186/1471-2334-11-35

5. Latimer RL, Shilling HS, Vodstrcil LA, Machalek DA, Fairley CK, Chow EPF, Read TR, Bradshaw CS (2020) Prevalence of Mycoplasma genitalium by anatomical site in men who have sex with men: a systematic review and meta-analysis. Sex Transm Infect epub date 2020/04/29. https://doi.org/10.1136/sextrans2019-054310

6. Bignell C, Unemo M (2013) 2012 European guideline on the diagnosis and treatment of gonorrhoea in adults. Int J STD AIDS 24:8592. https://doi.org/10.1177/0956462412472837

7. Hadgu A (1996) The discrepancy in discrepant analysis. Lancet 348(9027):592-593. https://doi.org/10.1016/s0140-6736(96) $05122-7$
8. Hadgu A, Dendukuri N, Wang L (2012) Evaluation of screening tests for detecting Chlamydia trachomatis: bias associated with the patient-infected-status algorithm. Epidemiology 23:72-82. https:// doi.org/10.1097/EDE.0b013e31823b506b

9. Lewis N, Dube G, Carter C, Pitt R, Alexander S, Ison CA, Harding J, Brown L, Fryer J, Hodson J, Ross J (2012) Chlamydia and gonorrhoea contamination of clinic surfaces. Sex Transm Infect 88:418-421. https://doi.org/10.1136/sextrans-2012-050543

10. Meader E, Waters J, Sillis M (2008) Chlamydia trachomatis RNA in the environment: is there potential for false-positive nucleic acid amplification test results? Sex Transm Infect 84:107-110. https:// doi.org/10.1136/sti.2007.027862

11. Toepfer M, Herrmann B, Sansone M, Lilja C, Nolskog P (2020) Environmental contamination by Chlamydia trachomatis RNA can cause false-positive test results in clinical samples. Sex Transm Dis. https://doi.org/10.1097/OLQ.0000000000001323

12. Unemo M, Seifert HS, Hook EW 3rd, Hawkes S, Ndowa F, Dillon JR (2019) Gonorrhoea. Nat Rev Dis Primers 5:79. https://doi.org/ 10.1038/s41572-019-0128-6

13. Field N, Clifton S, Alexander S, Ison CA, Hughes G, Beddows S, Tanton C, Soldan K, Coelho da Silva F, Mercer CH, Wellings K, Johnson AM, Sonnenberg P (2015) Confirmatory assays are essential when using molecular testing for Neisseria gonorrhoeae in lowprevalence settings: insights from the third National Survey of Sexual Attitudes and Lifestyles (Natsal-3). Sex Transm Infect 91: 338-341. https://doi.org/10.1136/sextrans-2014-051850

Publisher's note Springer Nature remains neutral with regard to jurisdictional claims in published maps and institutional affiliations. 